\title{
Egypt: Expand access to postabortion care
}

Frontiers in Reproductive Health

Follow this and additional works at: https://knowledgecommons.popcouncil.org/departments_sbsr-rh

Part of the International Public Health Commons, Public Health Education and Promotion Commons, and the Women's Health Commons

How does access to this work benefit you? Let us know!

\section{Recommended Citation}

"Egypt: Expand access to postabortion care," FRONTIERS OR Summary. Washington, DC: Population Council, 2000. 


\title{
Egypt Postabortion \\ Care \\ Expand Access to Postabortion Care
}

OR Summary 12

\begin{abstract}
Training providers and introducing a case management protocol led to improved postabortion care at 10 government and teaching hospitals in Egypt. Patients reported shorter waits and greater satisfaction with the medical services they received. Physicians adopted treatment methods associated with fewer complications and provided more health-related information to patients.
\end{abstract}

\section{Background}

The Population Council has supported a series of studies to improve the quality of postabortion care (PAC) in Egypt. A 1994 pilot study in two Egyptian hospitals showed that upgrading PAC and training physicians in manual vacuum aspiration (MVA), infection control and counseling led to significant improvements in the care of postabortion patients. Studies have shown that MVA with local anesthesia is associated with lower complication rates and shorter patient stays than sharp curettage with general anesthesia.

This 1997 study, conducted by the Egyptian Fertility Care Society with support from the Population Council, sought to institutionalize improved postabortion medical care and counseling procedures in ten hospitals - seven government hospitals and three university hospitals. Five senior physicians from each hospital attended a five-day training course in MVA, infection control, and family planning (FP) counseling. The physicians then supervised four months of on-the-job training of doctors and nurses at the 10 hospitals. A case management protocol, including emergency medical treatment, pain control, and FP counseling, was also introduced.
Researchers measured the resulting changes in knowledge and practice by administering surveys before (255 physicians, 311 nurses, and 508 patients) and after (246 physicians, 263 nurses and 497 patients) the intervention. Data were also collected from 1,036 medical records.

\section{Findings}

- After their training physicians had significant gains in knowledge about short-term complications, adverse health impacts of unsafe abortion, and the immediate return of fertility following postabortion treatment. Physicians were more likely after the intervention to recognize that PAC patients should receive information about the cause of miscarriage and the need to eat well, rest and use contraception during recovery.

\section{Care of Postabortion Patients}

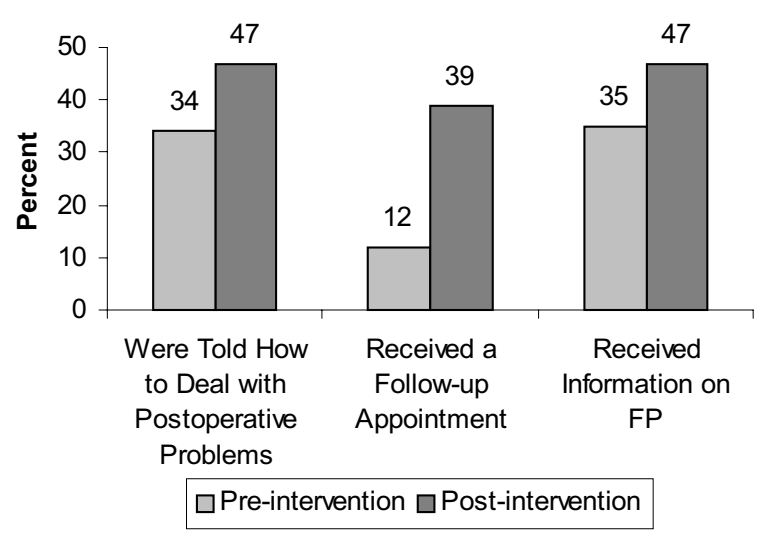


- After the intervention, physicians shifted from nearly universal use of dilatation and curettage (D\&C) to use of MVA in 57 percent of cases requiring emergency medical treatment. More than three in four physicians reported lower complication rates with MVA, compared with $\mathrm{D} \& \mathrm{C}$. More than half said that MVA is more effective and easier to use than D\&C.

- The shift to MVA led to a shift from general anesthesia to local anesthesia in about 30 percent of the cases. Following the intervention, the proportion of physicians stating that mild analgesia and local anesthesia can reduce patients' anxiety increased significantly. However, pain control techniques still need improvement:

18 percent of post-intervention patients did not receive any pain control medication. Although the proportion of patients reporting extreme pain did not increase significantly, reports of moderate pain increased five-fold (from $5 \%$ to $27 \%$ ).

- Nearly three in four (73\%) of the postintervention PAC patients stated that the service they received at the hospital was excellent, compared with 44 percent before the intervention. Post-intervention patients were more likely than pre-intervention patients to report that the provider was friendly, that they waited less than 30 minutes for medical services, and that they received information about possible complications, their management, and follow-up.

- The proportion of PAC patients who said that they had received family planning information at the hospital increased from 35 percent to 47 percent. However, only 7 percent of the PAC patients received a contraceptive method before discharge.

\section{Policy Implications}

- Training and protocols for PAC, including procedures for control of pain and infection and family planning counseling and services, should be standardized in hospitals as well as undergraduate and graduate medical schools. Government and teaching hospitals should include MVA supplies as standard items in their budget and should ensure an adequate supply of pain medication.

- Some aspects of PAC still need improvement: pain control, information given to patients, and the provision of FP counseling and services. Nurses should be given a greater role in comforting, counseling, and informing PAC patients. Simple guides on MVA instruments and FP counseling should be developed.

\section{Utilization}

- The Ministry of Health and Population's "Healthy Mother/Healthy Child" project is training providers in MVA and other elements of PAC, including pain control and referral to family planning services. Ten new hospitals have introduced improved PAC through the Healthy Mother/Healthy Child project-five in Aswan, two in Luxor, and three in South Qena. Expansion into new sites in Fayoum and Bani Sewef is underway.

Nawar, Laila et al., 1997. Scaling-up Improved Postabortion Care in Egypt: Introduction to University and Ministry of Health and Population Hospitals. For more information, contact: Population Council, 6A Mohamed Bahie Eddine Barakat St., $10^{\text {th }}$ floor, Giza, Egypt.Tel.20-2-571-9252; Fax 20-2-570-1804; E-mail: frontiers@pccairo.org.

This project was conducted with support from the U.S. AGENCY FOR INTERNATIONAL DEVELOPMENT under Contract Numbers DPE-C-00-90-0002-10 and DPE-3030-Q-00-0023-00, and Cooperative Agreement Number HRN-A-00-98-0001200 . 